\title{
Charge Neutralization and Direct Thrust Measurements from Bipolar Pairs of Ionic- Electrospray Thrusters
}

Daniel G. Courtney* and Herbert Shea

Microsystems for Space Technologies Laboratory, Institute of Microengineering Neuchâtel campus, School of

Engineering, École Polytechnique Fédérale de Lausanne, Rue de la Maladière 71b, 2000 Neuchâtel, Switzerland

*dcourtney@alum.mit.edu

Kathe Dannenmayer and Alexandra Bulit

ESA-ESTEC, Noordwijk, Netherlands

Accepted for publication in the AIAA Journal of Spacecraft and Rockets, 2017

This is an author generated pre-print of the final (accepted) draft manuscript. 


\title{
Charge Neutralization and Direct Thrust Measurements from Bipolar Pairs of Ionic-Electrospray Thrusters
}

\author{
Daniel G. Courtney* and Herbert Shea ${ }^{\dagger}$ \\ Microsystems for Space Technologies Laboratory (LMTS), \\ Ecole Polytechnique Federale de Lausanne (EPFL), Neuchatel, CH-2002, Switzerland \\ Kathe Dannenmayer ${ }^{\ddagger}$ and Alexandra Bulit ${ }^{\S}$ \\ ESA-ESTEC, Noordwijk Netherlands
}

\begin{abstract}
Electrospray (ES) thrusters are unique in their ability to emit both positive and negative beams, each contributing similarly to thrust. Spacecraft charging could be prevented without a dedicated neutralizer if currents of simultaneously emitted opposing polarity beams were matched; despite a lack of implicit coupling between them. We discuss and evaluate experimentally both an active current-balance control circuit and a passive self-balancing configuration. Our highly ionic ES source includes a pair of machined porous glass ES emitter arrays in a single holder. In the passive configuration, unbalanced charge collection on a common floating extractor yields rapid charging towards artificially imposed $200 \mathrm{~V}$ limits without means for charge suppression, showing the unsuitability of that architecture. Active balancing of emitted currents was fully effective at charge neutralization during stable periods of emission, yet insufficient during potential alternations. We present methods to improve this approach, including demonstrating beam current modulation at up to 450 Hz. Direct thrust measurements when simultaneously emitting opposing polarity beams of the ionic liquid 1-ethyl-3-methylimidazolium tetrauoroborate are presented. Direct thrust measurements up to $35 \mu N$ were in excellent agreement with commanded levels and computations based on measured currents (up to $190 \mu A$ ) and voltages (up to $2100 \mathrm{~V}$ ).
\end{abstract}

\section{Nomenclature}

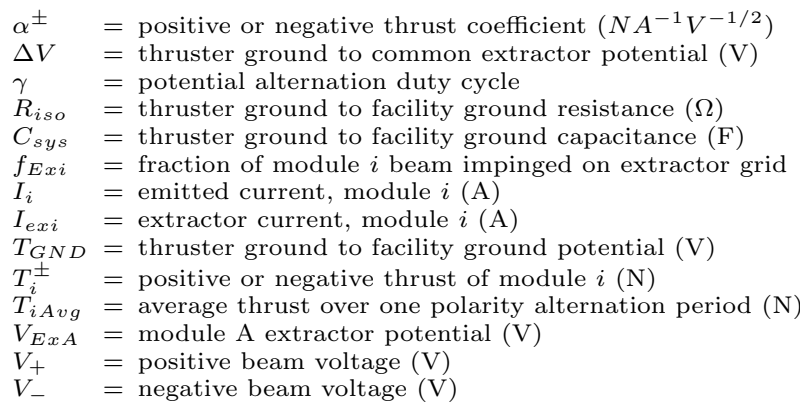

\section{INTRODUCTION}

Electrospray thrusters configured as Ionic Liquid Ion Sources (ILIS)[1], wherein beams of primarily ions and small clusters are emitted from Ionic Liquid (IL) propellants, have potential applications to both small

\footnotetext{
*Postdoctoral Researcher, EPFL-IMT-LMTS, AIAA Member, dcourtney@alum.mit.edu, Present address: Busek Co., Natick MA 01701 USA

${ }^{\dagger}$ Associate Professor, EPFL-IMT-LMTS.

${ }^{\ddagger}$ Electric Propulsion Engineer ESA-ESTEC-TEC-MPE.

$\S$ Electric Propulsion Engineer ESA-ESTEC-TEC-MPE.
}

satellites and as distributed thrusters on larger spacecraft. Compact thruster prototypes with 100's of emission sites distributed over a few $\mathrm{cm}^{2}$ frontal area have been shown to deliver 10's of $\mu N$ of thrust with less than $1 W$ of input power and with a specific impulse in the range of 1000 to $3000 s[2,3]$. The inherently low propellant flow rates are conducive to passive regulation of the IL propellant supply by capillarity; thereby obviating the need for active propellant control[1]. The ability to emit high performance beams of both positively and negatively charged particles has been shown previously, see for example [1-6]. The possibility of achieving charge neutralization without a dedicated neutralizer has, correspondingly, been envisioned[7, 8]. Removing the resources required for an electron emitting neutralizer would further simplify the technology and therefore suitability for small spacecraft.

Figure 1 presents a schematic overview of a chargeneutralized bipolar ILIS thruster pair. Equal beams of positive and negative ion beams are emitted simultaneously from adjacent sources due to enforced beam $\left(V_{A}, V_{B}\right)$ and extractor $\left(V_{E x A}, V_{E x B}\right)$ voltages. Periodic potential alternation can be employed to suppress electrochemical degradation within the IL propellant and at contact electrodes $[9,10]$. Here we discuss the viability and some subtleties of simultaneous bipolar emission and present charge neutralization and direct thrust measurements from demonstration devices, similar to those presented in Ref. [2]. 


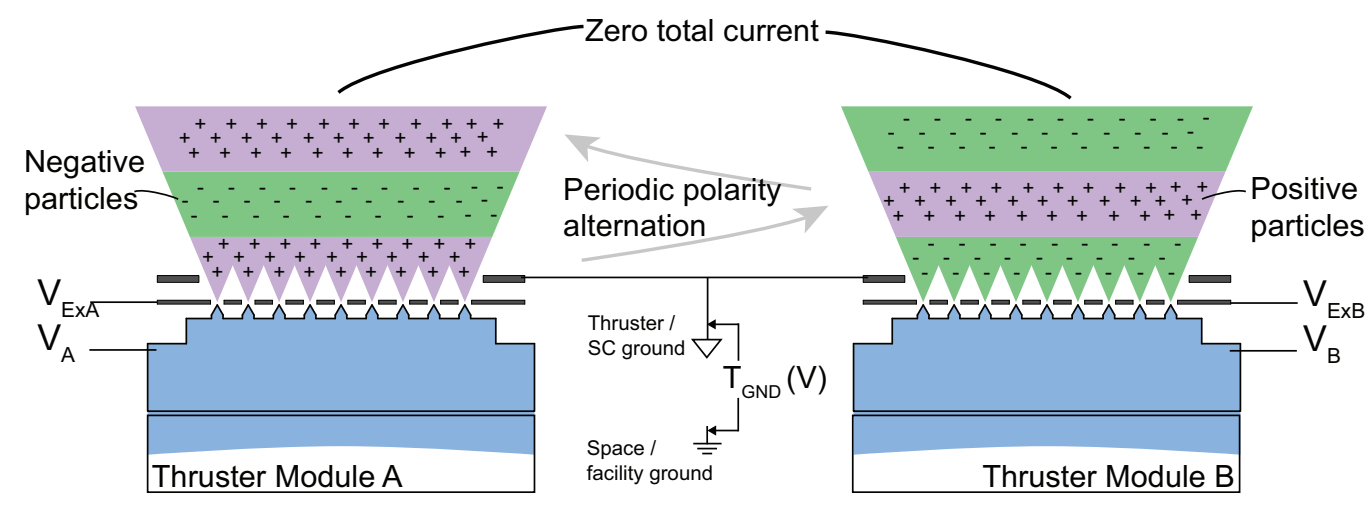

FIG. 1: Electrospray sources configured to emit zero total beam current through simultaneous bipolar emissions.

\subsection{ILIS Charge Neutralization}

Electrospray and liquid metal Field Effect Electrospray (FEEP) thrusters do not emit electrons inherently. They are often (necessarily in the case of FEEP) operated at only positive polarity alongside a neutralizing electron source[11-15]. As a particularly relevant example, the NASA-JPL Drag Reduction System (DRS) recently flown aboard the ESA led LISA Pathfinder mission demonstrated colloidal electrospray thrusters. This system[13], the first demonstration of electrospray propulsion in space to be reported in detail[14], includes a carbon nanotube field effect cathode as neutralizer.

Here, we primarily consider charge neutralization as opposed to beam neutralization. Charge neutralization requires the total emitted and collected currents from a spacecraft or electrically isolated thruster module to sum to zero to prevent charging. Without neutralization, a spacecraft's potential relative to its environment could quickly charge, leading to issues including damaging arcs or discharges, corruption of scientific measurements and/or a disruption of the emitted beam[16, 17]. Detrimental effects may occur due to both differential charging between electrically isolated components[18] or charging of the spacecraft and as a whole with respect to the ambient space plasma[16]. When ion beams are emitted, charging to a potential approaching the beam energy could stall the emission and attract particles back towards the spacecraft[19]. In the dual ion beam configuration at hand it is noteworthy that while this would lead to an attraction of ions at one polarity, the opposite polarity would be further accelerated away from the spacecraft[20].

The degree to which a spacecraft may float at high potential, and the effects of such potentials, are heavily dependent on the local environment and spacecraft design. Environment induced current sources may include the local plasma and/or photo-emission from spacecraft surfaces. ILIS and colloidal electrospray thrusters are highly scalable (see for example Refs. $[3,4,6,21])$ yet can also be configured to provide ex- treme levels of precision[13]. Accordingly these systems are applicable to a myriad of applications both at Low Earth Orbit (LEO) and in higher orbits including GEO or interplanetary space.

An immediate complication inherent to ion-ion charge neutralization using ILIS is evident considering applications at high orbits. Here, the plasma density is low and therefore the Debye length $\lambda$ will be much larger than a small satellite such that electron/ion collection from ambient may be negligible. When $\lambda$ is relatively large, the capacitance of an approximately spherical spacecraft of radius $R_{s c}$ is shown by Hutchinson[22] to be $C_{s c} \approx 4 \pi \epsilon_{0} R_{s c}\left(R_{s c} / \lambda+1\right)$, reducing to simply the vacuum level $4 \pi \epsilon_{0} R_{s c}$ when $R_{s c}<<\lambda$. In this limit, a $30 \mathrm{~cm}$ radius small satellite would have capacitance $C_{s c} \approx 30 p F$ and a current imbalance of only $10 \mu \mathrm{A}$ would charge a spacecraft to $1 k V$ in only $3 \mathrm{~ms}$. Thus, practical beam current equalization must occur quickly and to a high degree of accuracy. High voltage charging in GEO may not necessarily be catastrophic, see for example the discussions of the SCATHA spacecraft in Refs. [17, 19, 23]. However; beam stalling complications such as sputter damage by returned ions or failures due to differential charging may be incurred[19].

In the relatively dense LEO plasma, small Debye lengths augment the effective capacitance of the spacecraft and a relatively large level of ambient electron flux may be accessible from the local plasma with minimal potential rise. However; the level of collected current is highly variable. The reviews of Hastings[23] and Garrett[18] in particular detail conditions whereby significant spacecraft charging may occur in LEO; with specific emphasis on the drastic reductions in local plasma density which can occur in eclipse or in nearpolar orbits. A particular example, highlighted by both reviews is evidence that the Defense Meterological Satellite Program (DMSP) satellites charged to more than $-1 k V$ in their sun-synchronous orbits, see Ref. [24] for details. Suppression of negative charging is particularly challenging[16, 23] as the ambient ion flux, necessary to suppress negative charge, is much lower than electron flux and predominantly only collected by 
ram facing surfaces. In addition to governing collected ion flux, spacecraft attitude may also influence electron collection due to wake effects[23] which limit current collection to conducting surfaces in LEO. For example, the space shuttle charged positively to $5 k V$ by ejection of a $(5 \mathrm{kV})$ electron beam when the shuttle's primary current collection surfaces (engine bells) were in the spacecraft's wake during STS-9[25].

Hence, while some circumstances may exist where rapid swings in spacecraft charge may be acceptable or be suppressed by the local environment, a bipolar ILIS system should, in general, be capable of rapid charge neutralization to maximize applicability. Plasma thrusters that emit a bipolar beam naturally or use plasma based cathode neutralizers, such as hollow cathodes, are inherently forms of plasma contactors which automatically provide a passive means of charge neutralization[23]. Here, the current of low energy electrons within the emitted plasma is not directly controlled but rather self regulates due to the changing potential structure within the beam and/or downstream of emission. The result is an automatic charge balance facilitated by a coupling voltage between the cathode, thruster plume and local space plasma of a few 10's of volts[26]. This scenario differs from bipolar operation of an ILIS thruster where positive and negative high particle mass, high energy beams are emitted without any low energy electron population. Currents of these two beam are dictated by the applied electrostatic fields in the vicinity of each source alone (in passively fed thrusters), with no implicit coupling between those fields. If beam currents were well balanced as part of the thruster design, spacecraft charging could be held close to the natural level reached by environmentally induced collection/emission of charged particles from spacecraft surfaces.

Two methods of enforcing automatic charge neutralization are experimentally evaluated herein: i) a passive method previously proposed and tested in Refs. [3, 20] and ii) a closed loop active current-control method.

\subsection{Beam Neutralization}

Beam neutralization refers to the neutralization of space-charge within the emitted beam(s). Beam neutralization can contribute to performance through, for example, influencing the aforementioned cathode to plume coupling voltage in plasma-based electrostatic thrusters, or contribute to accelerating low energy (e.g. charge-exchanged) particles within a plume back towards the spacecraft. The latter may lead to sputtering or contamination damage on spacecraft surfaces $[23,26]$. In ILIS, the extremely low vapour pressure of ILs limit emissions to species ejected in the electrospray. Accordingly nearly all emitted particles travel at high speed, suppressing the degree to which charge-exchange ions may be readily attracted back to weakly charged spacecraft surfaces. Furthermore, due the low plume density, high particle mass and high energy of bipolar ILIS beams, interactions due to poor beam neutralization are not expected to significantly alter thruster performance if charge neutralization is achieved. In section 3.3 we present thrust measurements from a grounded device emitting adjacent actively balanced bipolar beams and compare those measurements with a sum of calculated thrusts based on previously published correlations.

\section{EXPERIMENT APPARATUS AND METHODS}

\subsection{Sources}

A demonstration thruster comprising two of the sources described in Ref. [2] within a single $57 \mathrm{~mm}$ x $27 \mathrm{~mm} \times 10 \mathrm{~mm}$ package was used in all bipolar measurements presented, see Figure 2. As in the cited work, each emission source (module) contains a $1 \mathrm{~cm}$ diameter, $3 \mathrm{~mm}$ thick porous borosilicate substrate featuring nine $7.5 \mathrm{~mm}$ long triangular prisms each terminated by a sharp (few 10's of $\mu m$ radius) edge. An extracting grid comprising $350 \mu \mathrm{m}$ wide laser cut slits in $100 \mu \mathrm{m}$ thick molybdenum is aligned with the emitting edges and positioned roughly $100 \mu \mathrm{m}$ above them. $\mathrm{A} \sim \pm 2 k V$ voltage applied between the grid and IL soaked within the porous glass initiates numerous emission sites along the edges, facilitating ion beams of several hundred $\mu A$. Unlike in Ref. [2], contact with the IL is made via a stainless steel, rather than aluminum, flange. The same conventional CNC milling technique was used to form the emitters. A second porous borosilicate disc (Duran Group P3 grade) was mounted below each emitting layer and served as the IL reservoir. Since the IL itself is polarized, independent reservoirs for each module were required. The interfacial Laplace pressure induced by the reservoir has been shown to be critical to the emission characteristics[27]; the P3 reservoir grade used in all experiments here matches Ref. [2].

All demonstration thrusters were wet with the IL 1-ethyl-3-methylimidazolium tetrafluoroborate (EMI$\mathrm{BF}_{4}$ ) which had been previously degassed in high vacuum for at least 12 hours. Liquid has been applied to the upper surface of each emitter and allowed to fill both the emitter and reservoir under vacuum. The extractor grid was aligned and fixed into position after wetting.

\subsection{Test Facilities}

The charging and current control experiments presented in section 3.1 and 3.2 have been performed primarily at the Microsystems for Space Technologies Laboratory (LMTS) at the Ecole Polytechnique Federale de Lausanne, Switzerland. Additionally, thrust measurements were made at the European Space Agency (ESA) Propulsion Laboratory (EPL) at the European Space 


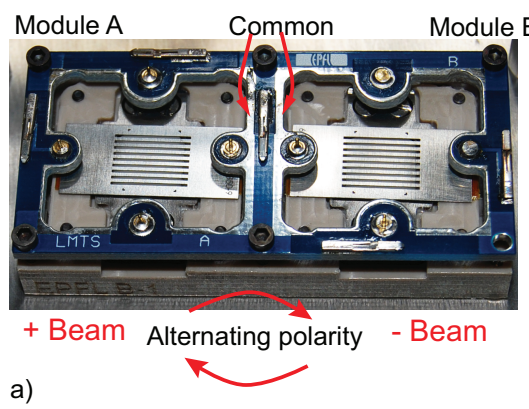

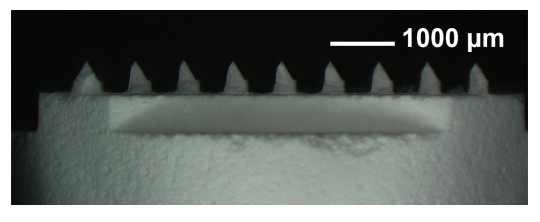

b)

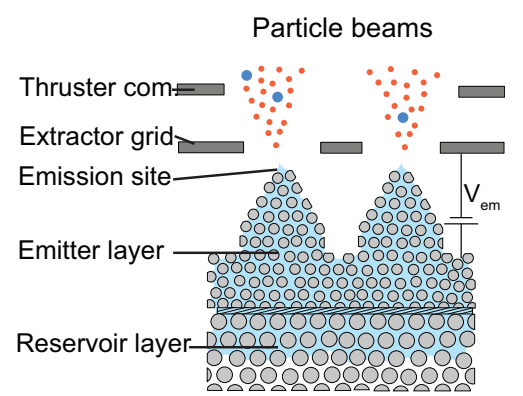

c)

FIG. 2: a)Photograph of a dual thruster module device. b) Microscopic photograph of the porous glass emitters applied here. c) Cross-sectional overview of the critical components of each thruster module.

Research and Technology Centre (ESTEC), Noordwijk, Netherlands.

At the EPFL, the facility comprised a six-way ISO160 cross pumped by a Varian V70 turbomolecular pump. The measured pressure typically rose from a base pressure less than $2 \times 10^{-} 6$ mbar to, at most, $1 \times$ $10^{-5}$ mbar during emission. At ESTEC, measurements were performed within the Galileo vacuum facility comprising a $1.0 \mathrm{~m}$ long $\mathrm{x} 1.2 \mathrm{~m}$ diameter chamber pumped by both a turbomolecular pump and cryo-heads yielding base pressures below $1 \times 10^{-7}$ mbar and operational pressures up to $\sim 5 \times 10^{-6}$ mbar during emission.

In all presented data the emission polarity of each module has been alternated using a custom-made switching circuit driven by a $1 \mathrm{~Hz}$ square wave input with $50 \%$ duty cycle. This circuit utilized Voltage Multiplier OC100G optodiodes to effect optically-isolated symmetric switching of HV signals in less than $400 \mu \mathrm{s}$. In all tests one positive and one negative high voltage supply were used to power either one or two active modules. At the EPFL, EMCO H30NR and H30PR DC-DC converters were used to supply floating emission voltages during charge neutralization testing while Stanford Research Systems P350 high-voltage supplies were used during current following demonstrations. At ESTEC, FUG models HCN 140-12500 and HCN 35M20000 high voltage supplies were used as positive and negative supplies respectively.

Measurements of the emitter and extractor currents for both modules were made with custom made optically isolated current monitors. The accuracy of these monitors, verified with a Keithley 6487 picoammeter was within $\pm 2 \mu A$ at the currents of interest. As discussed in the introduction a few $\mu A$ of unbalanced current may be sufficient to induce relatively severe spacecraft charging and, as will be shown in section 3, was sufficient to induce charging on the isolated facility during neutralization tests. As a result, this level of accuracy presents some limit to quantitative interpretation of current sums when near zero. The current monitor internal circuitry includes a $1 \mathrm{kHz}$ low pass filter.

During charge neutralization measurements, all input and output signals were isolated via opto-isolators or disconnected. Two zener diodes were installed between the local reference ground and facility ground to limit charging of the equipment to within $\pm 200 \mathrm{~V}$. The effective capacitance and resistance of the local reference to facility isolation were measured to be less than $1 n F$ and approximately $500 M \Omega$ with an Agilent E4980A LCR meter and a Keithley 6487 electrometer respectively. The thruster reference to facility ground potential $T_{G N D}$ was measured using a non-inverting amplifier a gain of 100:1. The available facilities did not support active measurement of true beam voltage outputs during floating operation due to a lack of isolated feedback. Instead, characterizing current versus voltage measurements were made prior to tests with the facility and local reference grounds connected.

It should be noted that facility effects may have contributed to the measured data. For example, the relatively small vacuum chamber at EPFL was held at facility ground and would have emitted secondary electrons upon bombardment from the emitted electrospray beams. These secondary electrons could have suppressed charging, particularly to positive potential. However; on numerous occasions (see section 3) the thruster floating potential rose to large positive values.

Direct thrust measurements were made at the ESTEC facility using a Mettler-Toledo AX504 balance modified for use in vacuum. The balance output was verified using calibrated masses and had a resolution of approximately $1 \mu N$. An electromagnetic force actuator was used to verify consistent balance output periodically as the thruster was installed in the facility and electrical interconnects were made. Specifically, the relative response to consistent steps in magnetization current was measured with the thruster unloaded, with electrical connections made and, finally, with a moderate potential below the emission voltage applied. Thrust measurements were made after confirming relative responses in the final case matched the unloaded configuration. 


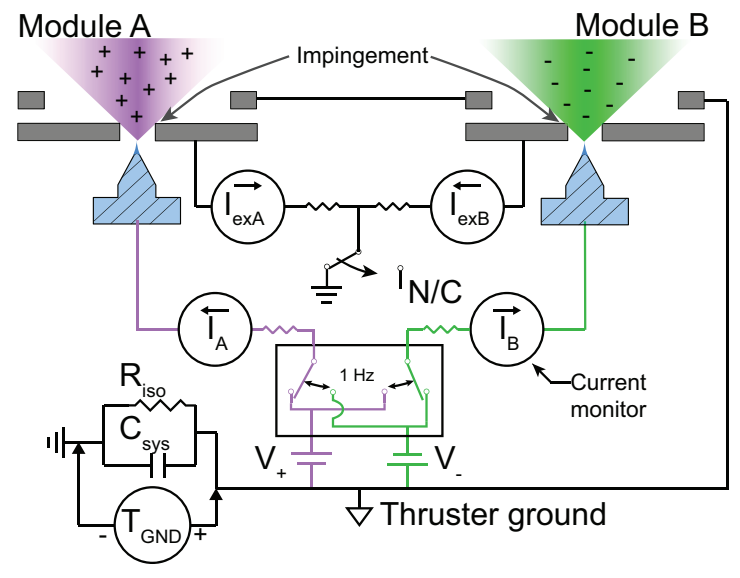

FIG. 3: Configuration for automatic charge neutralization with thruster supplies floated with respect to extractor electrodes at a common potential either floating or tied to facility ground.

\subsection{Passive Current Balancing}

Automatic current balancing has been proposed and recently demonstrated in Ref. [20] whereby the emitter supplies are floated with respect to a common extractor electrode. This configuration is shown in Figure 3. When in operation any excess charge accumulation at the thruster common due to a current imbalance leads to an increase or decrease in potential with respect to the common extractor. These variations in potential naturally tend to suppress or augment emissions in a manner which suppresses charge accumulation. That is, the extractor is intended naturally to float, relative to the thruster common, at a potential which enforces a current balance. Some degree of beam interception on the extractor is expected in any electrospray thruster; hence the impact of this charge accumulation was of particular interest here. Accordingly, two versions of this configuration were investigated in the work presented here. First, the common extractor was tied to facility ground. Second, the extractor has been isolated from the facility ground to emulate spacecraft conditions where, for example, the extractor may be tied to the spacecraft and have no active means for dissipating charge. Referring to Figure 3 these two configurations are denoted by a selectable extractor contact.

\subsection{Closed Loop Current Control}

An active feed back mechanism was experimentally evaluated in this study as an alternative to the passive balance method described above. Referring to Figure 4 , the potential between the IL and the extractor grid of module (A) was modulated by altering the extractor potential relative to the thruster ground $\left(V_{E x A}\right)$. The control circuitry permits $V_{E x A}$, and thereby the

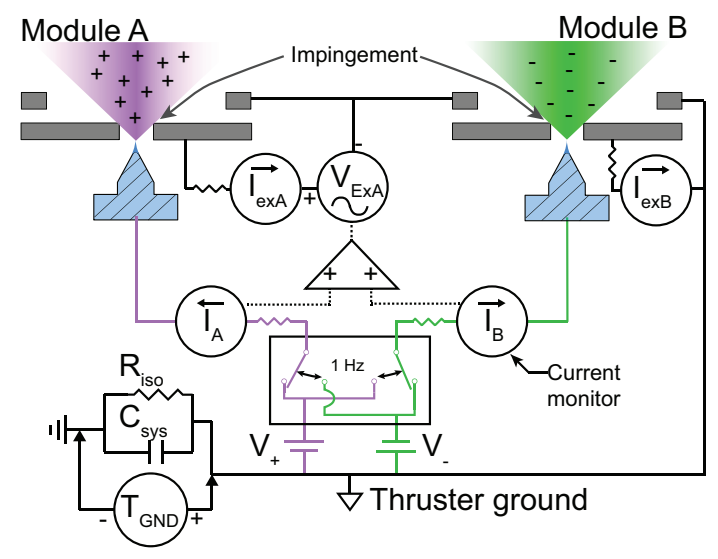

FIG. 4: Configuration for closed loop charge neutralization where the sum of emitted currents has been used to modulate the module A emitter to extractor potential.

emitter to extractor potential, to vary by up to the \pm $300 \mathrm{~V}$ limits of an EMCO G06CTR bipolar DC to DC converter. As will be shown in section 3, this range of extracting potential variation corresponds to many 10 's of $\mu A$ of current control.

The extractor voltage control circuitry is shown in Figure 5. Vishay LH1500AT solid state relays (SSRs) are controlled via a comparison of drive and follower inputs. The output is fed through a low pass filter (LPF in the Figure) with cut off frequency of approximately $300 \mathrm{~Hz}$. Although relatively slow compared with the charge rates described in the introduction, this cut-off frequency was selected to be compatible with the few $m s$ rise time characteristics of the SSRs. When used to balance current output, the comparator inputs comprise the module B current and the inverse of the module A current. The circuit drives the extractor voltage to be more positive when the comparator input is positive and negative otherwise. Hence when the drive signal (e.g. module B emitted current) is larger than the follower (e.g. inverted module A emitted current) the extracting potential at module $\mathrm{A}, V_{A}-V_{E x A}$ is reduced, promoting either enhanced negative emission or reduced positive emission; always tending towards a current that balances the drive signal. The follower signal could be amplified or reduced by a small amount prior to comparison with the drive via a configurable gain in a nominally unity-gain inverting amplifier. This manual tuning function has been included to accommodate for slight differences in the current monitor gains and, in practice, differences in the transmission fractions between devices. This component is not shown in Figure 5.

The extractor voltage, rather than that of the emitter, has been modulated for two largely practical reasons. First, electronic components and circuitry are simplified when controlled signals are at several hundred rather than several thousand volts. In ILIS, the 


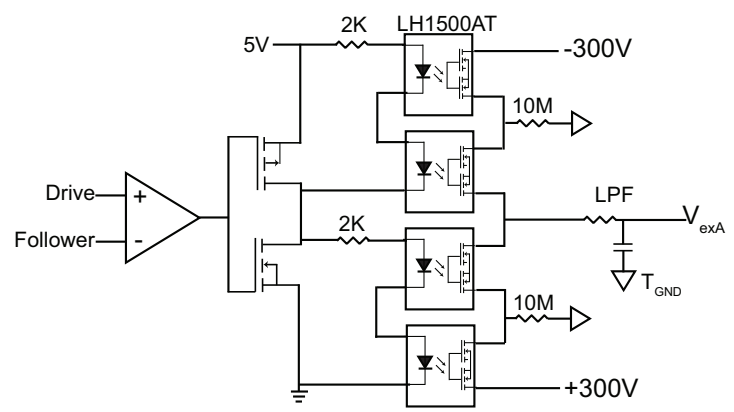

FIG. 5: Schematic overview of the balance driver circuit used here.

beam potential may be many $k V$ in some applications yet the operating voltage range is typically within several hundred volts of a start-up potential; below which no emission occurs (see the current-voltage measurements in section 3 ). Furthermore, as increasingly scaled up ILIS thrusters are envisioned, complications may arise when attempting to modulate the primary current carrying $\mathrm{HV}$ signals to the emitters. Under nominal conditions, the extractor electrode intercepts only a small portion of the emitted beam current making it further suited to modulation via low current components. Second, the emitter to ground plane potentials, $V_{A}$ and $V_{B}$ in Fig. 1, establish the total beam energies and therefore specific impulse and thrust-per-unitcurrent for constant species charge-to-mass ratio and beam shape. Through only modulating the extractor potential, the total beam energy and therefore these performance metrics are virtually unchanged. Nominally a second acceleration or deceleration grid aligned with and closely position downstream of each extractor orifice would ensure efficient acceleration or deceleration to the enforced outer potentials $[6]$ and provide a degree of performance-enhancing focusing when acceleration is applied. However, when in close proximity to the beam this electrode would present an additional source of beam current interception to be accounted for by a practical implementation of closed-loop totalcurrent control circuitry. In this demonstration, the extractor grid of module B was tied to thruster ground through a monitor and a simple ground plane on the connection PCB well beyond the plume serves to, albeit weakly, define the downstream ground plane of module A. This ground plane is identified as the 'Common' electrode in Fig. 2.

Current following data from an individual emitter module, emitting EMI- $\mathrm{BF}_{4}$, were acquired to validate and characterize the circuitry. In this test, a single source identical to that presented in Ref. [27] was utilized. This source again mirrors the configuration of Ref. [2] yet includes only a single $7.5 \mathrm{~mm}$ long emitter edge. Referring to Figure 6(a), two synchronized Agilent 33220A arbitrary function generators were used to supply a drive signal comprising a sinusoid modulating a $1 \mathrm{~Hz}$ square wave. The same $1 \mathrm{~Hz}$ square wave was used to alternate the emitter polarity. This artificial drive was input to the controller described above along with the emitted current feedback signal as the 'follower', see Figure 6(a). The frequency of the control signal was then varied while recording the emission current. Figure 6(b) provides sample data at $20 \mathrm{~Hz}, 50$ $\mathrm{Hz}$ and $100 \mathrm{~Hz}$ using a modulation amplitude of $\sim 5$ $\mu A$ about $\pm 25 \mu A$. At these relatively low frequencies reasonable current following was achieved. However, in Figure 6(c) data acquired at $150 \mathrm{~Hz}, 250 \mathrm{~Hz}$ and $400 \mathrm{~Hz}$ indicate the onset of both a phase lag and gain reduction. $\mathrm{A} \sim 2 \mu \mathrm{A}$ high frequency noise signal was present on all data and intrinsic to the current measurement circuit used in these circuit validation tests.

While short of a detailed spectrum analysis these data confirm that rapid modulation of the extractor grid provides a simple means for modulating the total emitted current at relatively high speed. However; the response rate fell below the $m s$ time scales discussed as being potentially required in the introduction. Despite their relatively slow speed, SSRs were selected in lieu of, for example, the OC100G optocouplers used in the switching circuit due to their low power consumption. The selected SSRs consume roughly $1 \mathrm{~mW}$ of power while in operation, while the OC100G optocouplers require in excess of $300 \mathrm{~mW}(100 \mathrm{~mA}$ at $3.0 \mathrm{~V}$ total forward voltage) when active. Compared with the few hundreds of $m W$ input to each thruster module, the latter is clearly significant hence the low power SRR option was favoured.

This SSR based circuit was utilized in all charge neutralization tests presented here. However; an additional single emitter current following test was performed using OC100G optocouplers to effect switching. That test, presented in section 4.2 indicated a cut-off frequency of $450 \mathrm{~Hz}$. The frequency limitations in Figure 6 may therefore have been predominantly limited by the drive circuitry rather than emission processes.

\subsection{Total Thrust Output from Bipolar Sources}

In earlier work[2] we demonstrated that thrust from the sources used here can be described by equation 1 for each polarity of emission. Equation 2 can then be applied to determine an average over one period of potential alternation.

$$
\begin{aligned}
& T_{i}^{ \pm}=\alpha^{ \pm}\left[\left|I_{\text {Beam }}\right| \sqrt{|V|}\right]_{i} \\
& T_{i \text { Avg }}=\gamma T_{i}^{+}+(1-\gamma) T_{i}^{-}
\end{aligned}
$$

Here, $T_{i}^{+}$and $T_{i}^{-}$are the thrust output from ILIS module $i$ (A or $\mathrm{B}$ ) in positive and negative polarities respectively. $I_{\text {Beam }}$ is the beam current, taken as the difference between emitted current and that intercepted by the extractor grid, while $V$ is the applied voltage and $\gamma$ is the alternation duty cycle. The terms $\alpha^{+}$and $\alpha^{-}$are aggregate coefficients accounting for the chargeto-mass ratio of emitted species, beam divergence and 


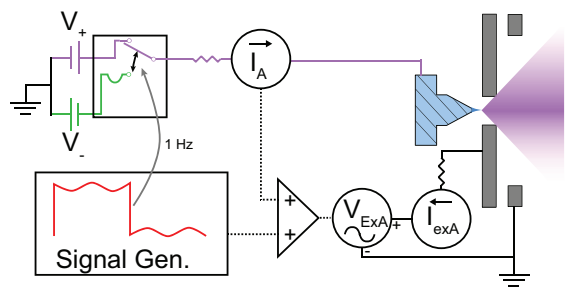

(a)

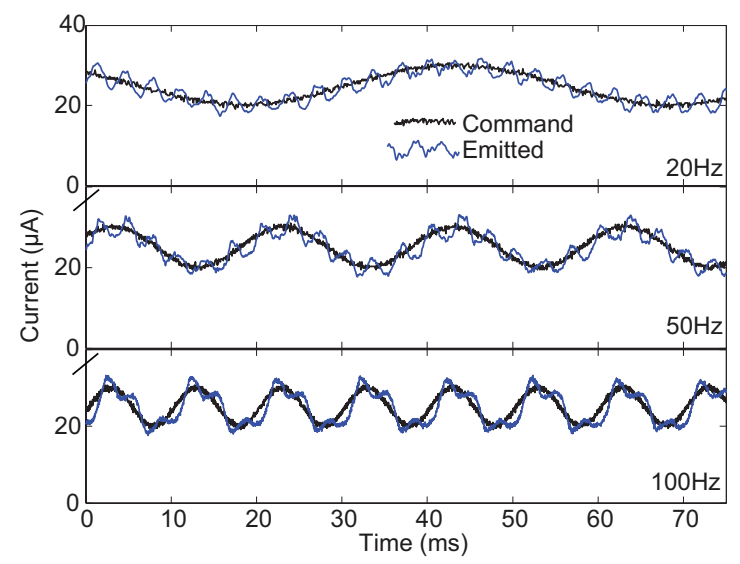

(b)

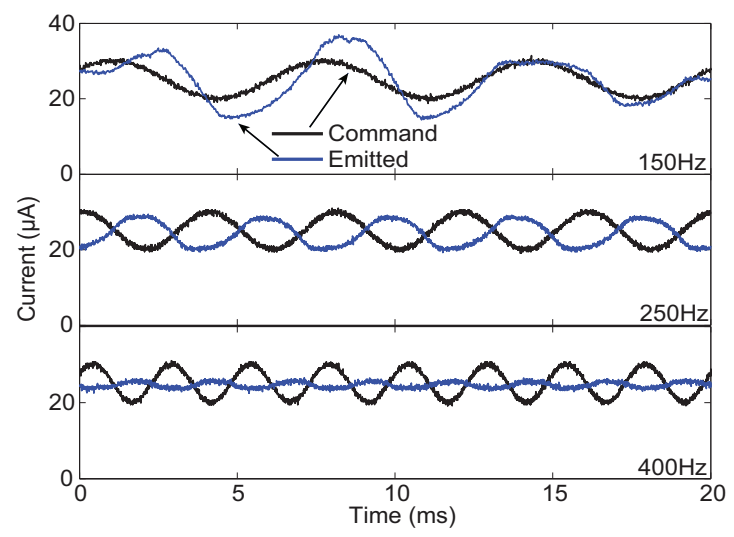

(c)

FIG. 6: Response of the current follower circuit to input sinusoidal waves at the indicated frequency emitting EMI-BF 4 .

energy losses. In Ref. [2] these were found to be $\alpha^{+}=$ $0.00201 N A^{-1} V^{-1 / 2}$ and $\alpha^{-}=0.00197 N A^{-1} V^{-1 / 2}$ when emitting EMI-BF $\mathrm{BF}_{4}$ from sources equivalent to those used here.

The form of equation 1 differs from that previously derived and reported for droplet dominated colloidal electrosprays[13] through the power of the current dependence. In colloidal devices, the charge-to-mass ratio and current are both strongly tied to an enforced mass flow rate[28], leading to charge-to-mass ratio which varies with current to the one-half power and ultimately a current to the three-halves dependence in expressions for thrust. However; in the presented devices, charge to mass ratio has been shown to be relatively constant over large ranges of beam current[2], leading to proportional contribution due to current in the thrust expression. Similarly, no clear trends in beam divergence or fractional energy loss over voltage have been observed

\section{RESULTS}

Current versus voltage measurements from the twin module device utilized in all charge neutralization results are shown in Figure 7. The relationships are similar to previously presented data from this type of emitter[2] and are characteristic of the emission from devices used for thrust measurements at ESTEC, presented at the end of this section. To highlight the many 10's of $\mu A$ discrepancies between current magnitudes without balance measures in place, the module A current data have been inverted and plotted alongside module B currents of the opposite polarity. Specifically, Figure 7(a) presents positive module B emission currents and (inverted) negative module $\mathrm{A}$ emission current. Figure 7(b) presents negative module B emission current and (inverted) positive module A current. After a startup region between roughly $1500 \mathrm{~V}$ and 1750 $V$ the currents are seen to rise at rates of, roughly, 0.6-0.7 $\mu \mathrm{A} / \mathrm{V}$. Hence the $\pm 300 \mathrm{~V}$ range of extraction voltage adjustment provided by the closed loop balance circuitry equates to up to $\pm 200 \mu A$ of current control. Beam interception by the extractor grid was typically a few to $\sim 10 \mu A$, equivalent to up to $\sim 5 \%$ of the emitted current.

Figure 8 presents unbalanced measurements of emitted current, the thruster common potential with respect to facility ground $\left(T_{G N D}\right)$ and the summation of all recorded currents. Here the thruster was configured as in Figure 4, yet with the balance circuit disabled such that the module A extractor was tied to $T_{G N D}$ through a current monitor. Sample data is provided over a $2 s$ period, yet the measurements are representative of the steady state in this configuration. As evident from Figs. 8a) and 8c), total emitted currents were generally non-zero in this configuration. Specifically Fig. $8 \mathrm{c}$ ) provides the summation of emitted and intercepted currents from both modules, the filtered trace has been smoothed with $10 \mathrm{~ms}$ moving average. Referring to Fig. $8 \mathrm{~b})$, when the total current was negative, the thruster common quickly charged positively until the $+200 \mathrm{~V}$ zener-diode enforced limit was reached. Similarly, during regions of net positive emission, the common potential charged negatively to the opposite limit. It is noteworthy that during the latter periods some coupling between modules may have been present as evidenced by the short period of relatively (but not completely) matched currents around $0 s$ and $1 s$ on the plot. The exact cause of this coupling was not deduced with certainty. 


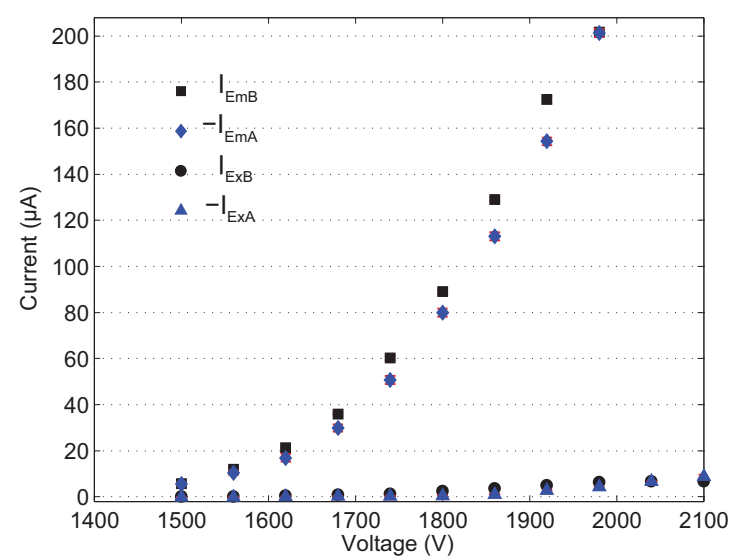

(a)Positive emission current from module B and (inverted) negative current from module A.

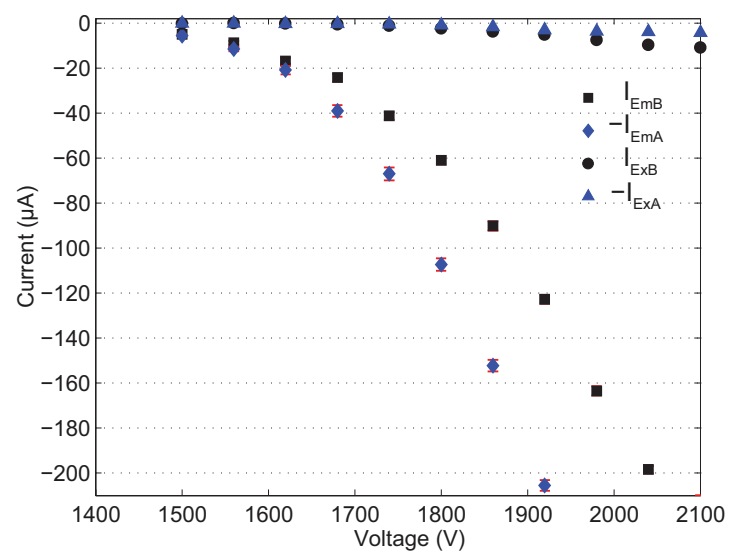

(b)Negative emission current from module B and (inverted) positive current from module A.

FIG. 7: Current versus voltage measurements. The module A current has been inverted to indicate typical levels of current magnitude mismatch during simultaneous \pm emissions.

\subsection{Passive Charge Neutralization}

The same device was subsequently arranged as in Figure 3 to evaluate automatic beam current balancing techniques. First, in Figure 9, the thruster power supplies and $T_{G N D}$ reference were floated with respect to the extractors. Both extractors were then tied to facility ground through their respective isolated current monitors. These data, presented in a format identical to Fig. 8, confirm that currents from the two emitters can be well balanced by this method as evidenced by the overlayed module B and negative module A currents in Fig. 9a). In Fig 9b) the thruster potential was observed to vary slightly, by roughly $20 \mathrm{~V}$ above or below the extractor potential (facility ground) in response to the emitted currents. Referring to Fig. 9c), the summation of current including extractor currents was close to zero considering the accumulated uncertainty

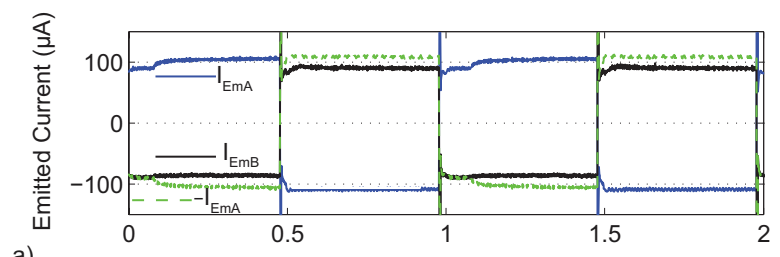

a)

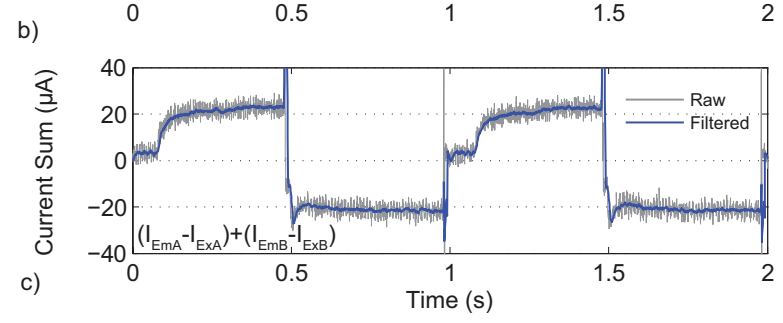

FIG. 8: Current, thruster common potential and current summation measurements under conditions of unbalanced emission.

of the monitors ( $\pm 2 \mu A$ per channel) yet a non zero trend remained; at roughly $+4 \mu A$ and $-5 \mu A$ during positive and negative emitter A current cycles respectively. When only the emitter current sum is considered (not shown) current summation magnitudes reduce to roughly $+0.5 \mu A$ and $-3 \mu A$ during positive and negative emitter A current cycles respectively; with propagated uncertainties of approximately $\pm 3 \mu \mathrm{A}$.

In Figure 10, the configuration again took the form of Figure 3 yet the common extractor electrode was left to float separately from the thruster power supplies. This configuration was intended to more accurately simulate a spacecraft environment through removing the path to facility through in the extractors. As evident from the sample data, the configuration is no longer stable. Note that in Fig. 10c) the vertical axis scale has been increased significantly compared with Figs. 8 and 9. In response to a small but non-zero current on the floating extractor electrodes the thruster common potential was observed to float up or down while maintaining an initial balance of currents similar to Fig. 9. Once the enforced $\pm 200 \mathrm{~V}$ limits were reached, and accordingly a path for current discharge from $T_{G N D}$ was established, the current outputs were no longer balanced in reaction to the charged extractor. It should be noted that the extractor currents, included in the current summation, lose some meaning here as there is initially no clear path to dissipate collected charge via the monitors. 


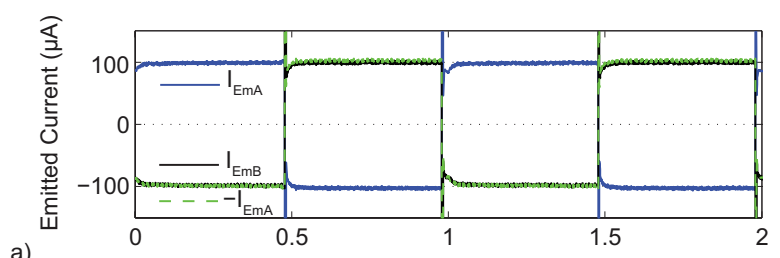

a)
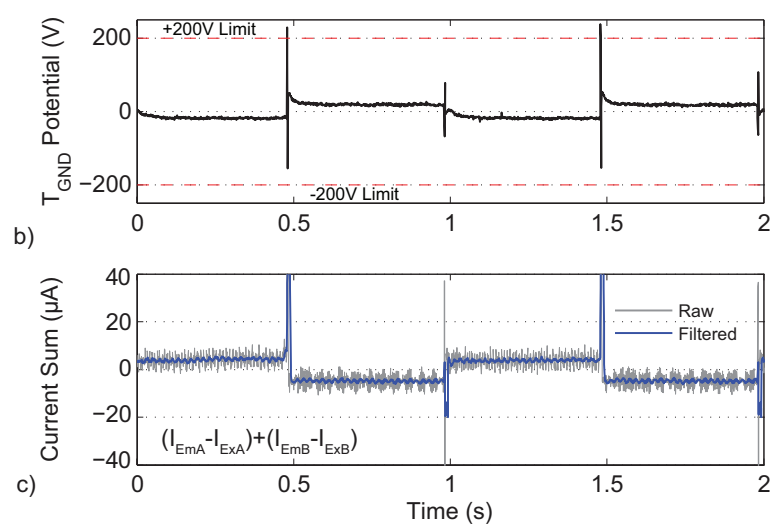

FIG. 9: Passively balanced sources with a common grounded extractor exhibited a low, but non-zero, thruster common potential and current summation.

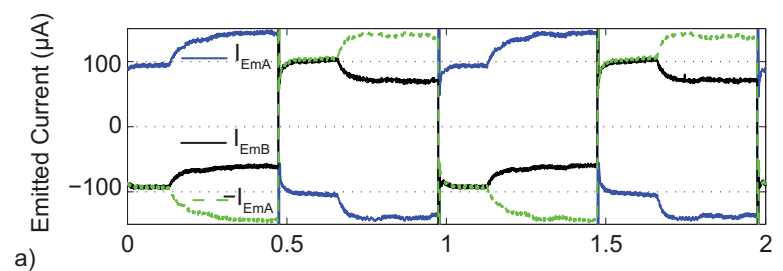

a)
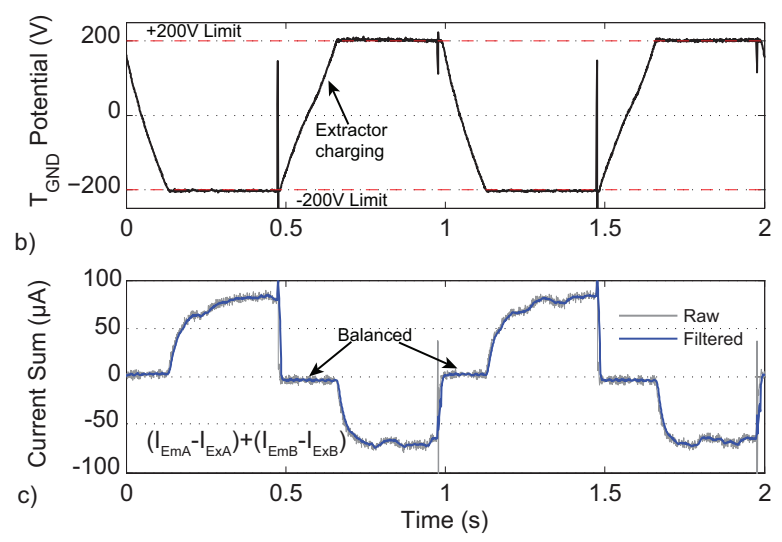

FIG. 10: Passively balanced sources with a common floating extractor exhibited current balancing briefly while the thruster common potential rose towards artificial $\pm 200 \mathrm{~V}$ limits.

\subsection{Closed Loop Charge Neutralization}

The same device used to obtain the data presented in Figures 8 through 10 was configured for active charge balancing as in Figure 4. Sample data are presented in Figures 11 and 12 . The thruster common potential $T_{G N D}$ is again indicated in Figs. 11b) and 12b) in addition to the potential applied to the extractor via the balancing circuit. Recall that the current balancing circuit employed in this study only considered the emitted current, not that the extractor grids. The manual tuning capability enabled some compensation for this omission through augmenting or decreasing the drive signal to the module A extractor electrode. In practice for a particular tuning setting, evidence of suppressed charging was only achieved in either the positive or negative emission mode from module B see Figs. 11 and 12 respectively. For example, in Fig. 11 total currents are largely nulled during positive beam emission from module B. In response, the $T_{G N D}$ potential rises only gradually before rapidly railing against the $-200 \mathrm{~V}$ limit upon the next polarity alternation cycle. In Fig. 12, the scenario is reversed in that the $T_{G N D}$ potential rises gradually off the $-200 \mathrm{~V}$ limit during periods of negative emission from module B. In these sample data currents are more globally matched compared with Fig. 11; however high speed transients during switching, poorly resolved by the $1 \mathrm{kHz}$ data acquisition, may have contributed to constraining the floating potential near the $-200 \mathrm{~V}$ limit.

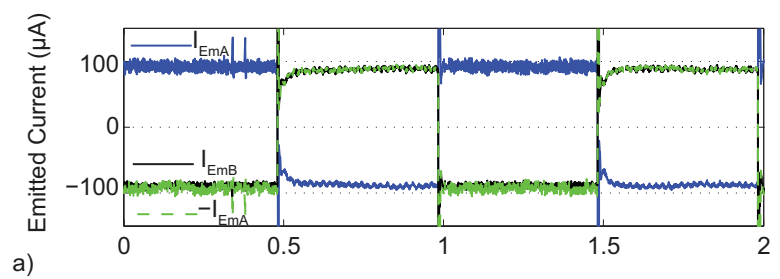

a)

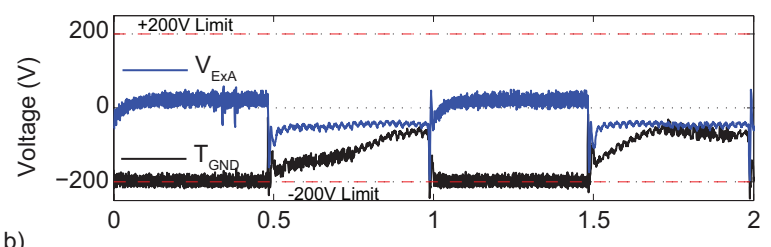

b)

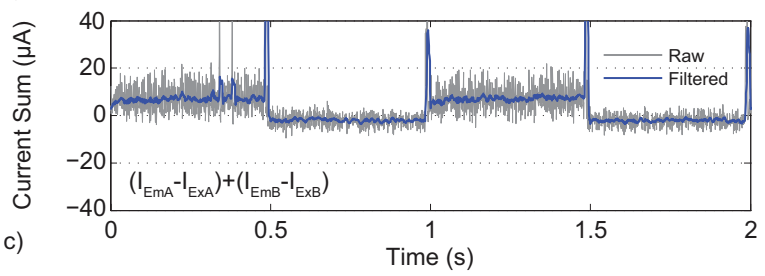

FIG. 11: Actively balanced currents tuned to suppress charging during positive B module emission but poorly tuned during the opposite half period of pulsation. 

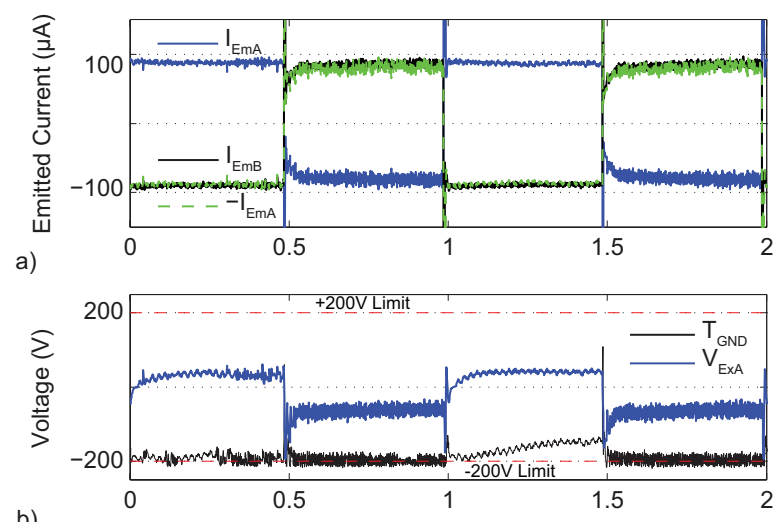

b)

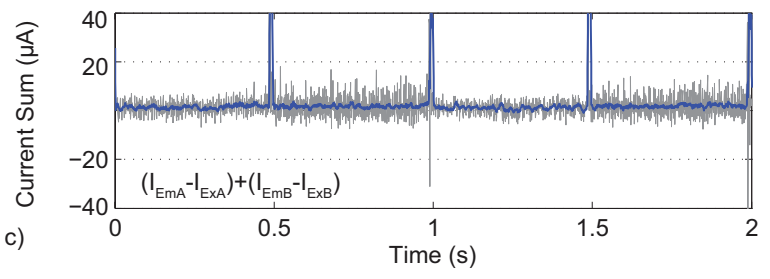

FIG. 12: Actively balanced currents tuned to suppress charging during negative B module emission but poorly tuned during the opposite half period of pulsation.

\subsection{Commanded Bipolar Thrust}

Thrust measurements were made during dual beam operation and with the current balancing circuit active. These data were acquired at the ESA ESTEC facility with a device that was nominally identical to that used to acquire the charging data presented in the previous sections. As in all previously presented measurements, polarities were alternated at $1 \mathrm{~Hz}$.

The software based closed loop control routine adjusted the power supply voltage settings in reaction to the error between desired thrust and the sum of that calculated using equation 2 for each module. In practice only the positive voltage was modulated through the proportional-integral controller while the negative supply magnitude was set to be $1 \%$ lower. This value was established empirically as suitable to minimize the difference in calculated thrust between periods of polarity alternation and thereby assist in reducing the spread of data in the directly measured thrust.

Figure 13 provides representative calculated and directly measured thrusts values over a sequence of commanded thrust measurements from 15 to $35 \mu N$ in 5 $\mu N$ steps. Per-module and total thrusts were calculated using equations 1 and 2 respectively. At peak thrust, of $\sim 35 \mu N$, each module was emitting approximately $\pm 190 \mu \mathrm{A}$ of current at $\pm 2100 \mathrm{~V}$; corresponding to $0.8 \mathrm{~W}$ of total input power. The zero level of direct thrust measurements has been adjusted for each pulse to account for drift in the balance output. Specifically, the zero level has been established from the average of data acquired both between $45 s$ and $15 s$ before and
$7 s$ and $45 s$ after each pulse. The measured thrust trace has been broken between each pulse to reflect this continuously adaptive zero reference. The calculated thrust data have been smoothed using a moving average filter time constant equivalent to $1 \mathrm{~s}$. Higher thrust levels were prohibited by practical current limits observed using OC100G optocoupler based polarity alternation circuitry. While recordings slightly below the commanded thrust level are evidence of deficiencies in the simplistic controller, good agreement between the calculated and directly measured thrust levels was observed over the full range tested.

\section{DISCUSSION}

\subsection{Passive Charge Neutralization}

Referring to section 3.1, we have identified charge collection on common extractor electrodes as a potential hindrance to passively achieving automatic charge neutralization on bipolar ILIS thrusters. Specifically, in Figure 9, balanced positive and negative currents were emitted in a stable manner from two modules when their extractor electrodes were at a common potential and tied to the facility ground. In that test, the floating potential of the source supplies deviated only slightly as needed to maintain balanced emitted currents. However; in Figure 10, when the tie to ground was severed, automatic current balancing was no longer stable and the supply common potential quickly railed against the imposed $\pm 200 \mathrm{~V}$ limits.

Referring to equation 3 and Fig. 3, this method of charge balancing relies on rapid adjustments to the thruster ground potential to common extractor potential difference, $\Delta V=T_{G N D}-V_{E x}$ due to net charge accumulation. Specifically, if a net positive emission occurs $\Delta V$ will charge negatively thereby suppressing positive emission and enhancing negative emission until an equilibrium between module $\mathrm{A}$ and $\mathrm{B}$ emitted currents $\left(I_{A}\right.$ and $\left.I_{B}\right)$ is quickly achieved. However; the charge collected at the extractor electrode must also be considered. For this electrode to remain neutral equation 4 must also be satisfied. Here $f_{E x A}$ and $f_{E x B}$ are the fractions of emitted current intercepted by the extractor grid. These interception fractions are uncontrolled characteristics of the particular thruster modules and hence $\Delta V$ is the only free variable in general. Thus a simultaneous solution to both Eqns. 3 and 4 at a single value of $\Delta V$ would be required in a stable configuration yet is generally not feasible. An exception exists if the intercepted current fractions are equal $f_{E x A}=f_{E x B}$. However; such a requirement imposes a severe constraint on device fabrication and operational consistency over time. This constraint was not achieved here. Hence the observed loss of control when the extractor current was floated in section 3.1 is consistent with an inability to simultaneously balance currents at the thruster common and extractor. Some mitigation could be imposed through adding capacitance between 


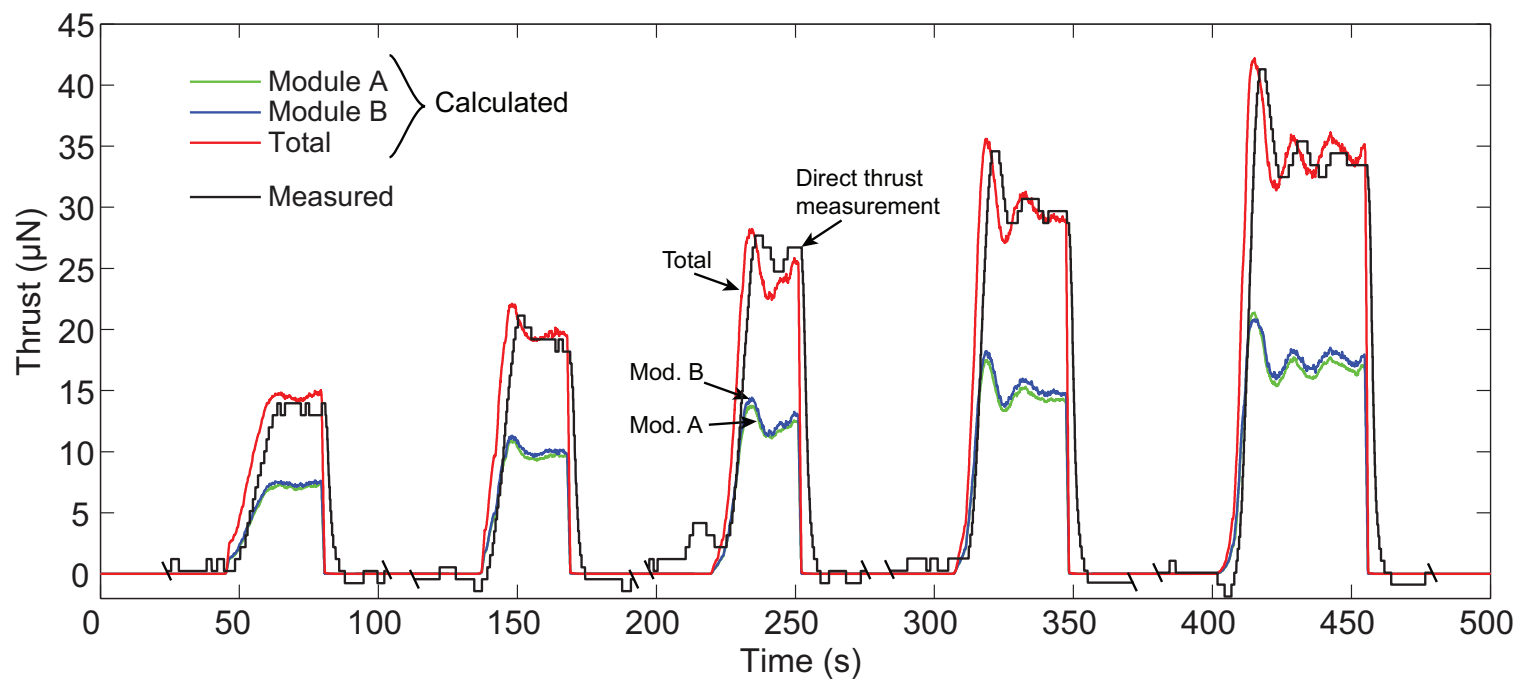

FIG. 13: Comparison of calculated and directly measured thrusts after repeated measurements.

thruster common and the extractor; however this would tend to suppress balancing rate using this method and again provide no explicit means to ensure overall charge neutralization is achieved.

$$
\begin{gathered}
I_{A}\left(V_{A}-\Delta V\right)+I_{B}\left(V_{B}-\Delta V\right)=0 \\
I_{E x A}\left(V_{A}-\Delta V\right)+I_{E x B}\left(V_{B}-\Delta V\right)=0 \\
f_{E x A} I_{A}\left(V_{A}-\Delta V\right)+f_{E x B} I_{B}\left(V_{B}-\Delta V\right)=0
\end{gathered}
$$

Ultimately, charge neutralization necessitates nullifying the sum of Eqns. 3 and 4; however as emission currents are only dictated by the local emitter to extractor potential differences, no feedback mechanism with respect to the local environment is implicit to enforce this constraint. If, for example, the extractor were tied to spacecraft chassis, this scenario could lead to a rapid accumulation of charge that may only be mitigated by either emitted particle return or environment dependent ambient particle fluxes or photo-emissions. While elegant in its simplicity, our finding has been that this passive method does not implicity guarantee total currents from the spacecraft sum to zero.

This result is consistent with that reported by MierHicks and Lozano[20]. There it was shown, theoretically and experimentally, that the return of, relatively, low energy particles in the emitted beam can suppress charging. Particles with energy well below the total beam potential are known to be present due to ion or ion-cluster fragmentation downstream of emission[29, 30]. While effective at maintaining charge neutralization to within several tens of percent of the beam voltage (positive or negative), those authors also demonstrated that acceleration of these heavy particles towards the thruster can induce damage. A potential risk to both the thruster and other spacecraft subcomponents.

\subsection{Active Charge Neutralization}

Closed loop current control is potentially less attractive in comparison to passive methods. However; the presented results in section 3.2, lend support to its applicability. In Figures 11 and 12 rapid potential swings from the imposed $-200 \mathrm{~V}$ limit were suppressed using closed loop control. However; manual tuning was required to achieve the states presented and no tuning setting could be achieved where the $T_{G N D}$ reference was steadily maintained near facility ground. Thus the sense and balance circuitry applied here did not completely null total currents over full periods of potential alternation. More sophisticated implementations of the circuit should include extractor currents and, if applicable, downstream accelerator grid currents in the summation to calculate and null total beam currents out of the spacecraft/thruster as accurately as possible.

The $\sim 100 \mathrm{~Hz}$ maximum operating frequency of the balance circuitry may not be sufficiently fast to suppress $m s$ timescale high voltage charging in some spacecraft environments, as discussed in the introduction. In Fig. 12, currents were largely matched save for possible brief periods during each potential alternation event. A higher bandwidth modulation circuit may be more appropriate to suppress such events. To consider if faster modulation rates may be feasible, an additional experiment was performed. Here the previously described current modulation circuit was modified to swap the Vishay LH1500AT SSRs for Voltage Multiplier OC100G optocouplers. The extractor modulation output low pass filter cut off frequency was also increased to approximately $1 \mathrm{kHz}$. Another single linear strip emitter configured as in Fig. 6(a) was used to evaluate this modification. This source was wet with the IL 1-ethyl-3-methylimadazolium thiocyanate (EMI-SCN).

Figure 14 presents $\mathrm{AC}$ modulation data with the 


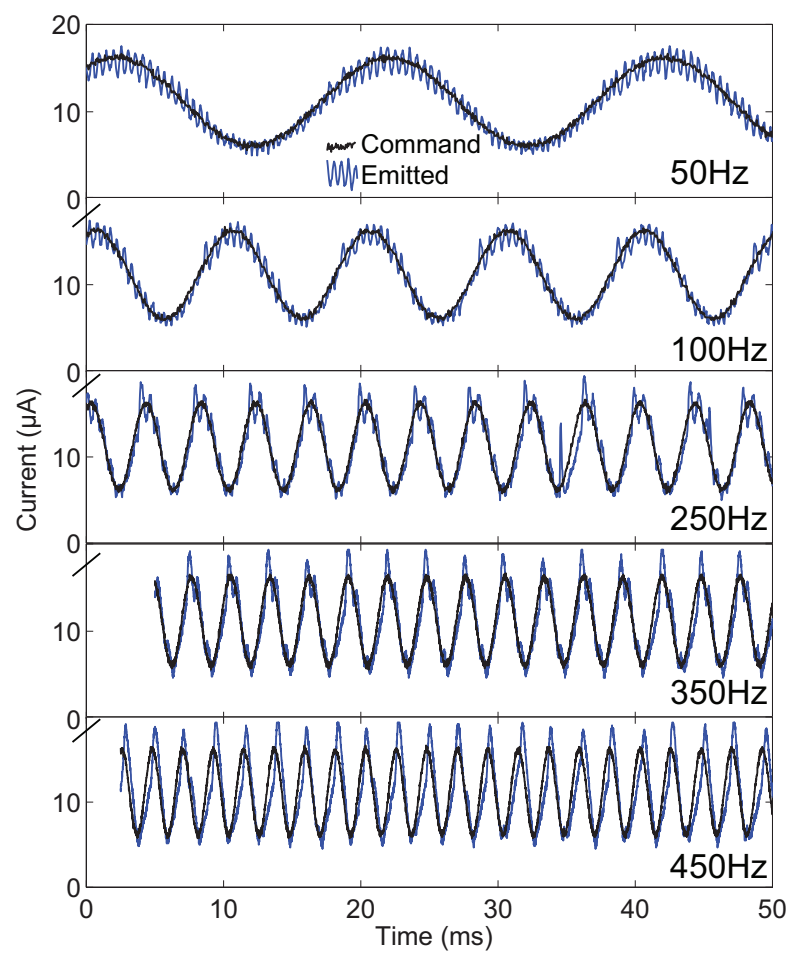

FIG. 14: AC drive tests with EMI-SCN, included to reinforce current timing limitations are, in part, due to electronics.

modified circuit and single emitter source. The drive signal was modulated between roughly 5 and $15 \mu \mathrm{A}$ of equivalent emission at the indicated frequencies. Current following was achieved at up to $450 \mathrm{~Hz}$ without loss of phase or attenuation, although an overshoot at the modulation maxima of a few $\mu A$ did persist at and above $250 \mathrm{~Hz}$. Compared with the SSR based circuitry, Fig. 6, these data demonstrate a more than two fold increase in supported frequency. If executed in a power efficient manner, modulation at these speeds may enable beam current matching at the $m s$ time scales necessary to suppress fast charging events on orbit. Such an implementation would also require accurate current sensing at a similar bandwidth. Circuitry capacitances could complicate this requirement through inducing current signals which are not associated with net charge loss, particularly during fast potential alternation transitions. Means to slow these transitions without losing the ability for rapid current balancing may be required. For example, transitions could comprise a slow modulation of the commanded beam voltages based on closed loop feedback of one current polarity while separately a fast current balance circuit, similar to that used here, acts to null total currents through modulating the opposing polarity's extraction voltage.

A physical time scale may limit modulation speed and, in particular, could compromise current matching during potential alternations. Lozano and Martinez-
Sanchez[31] measured onset delays of a few to 10's of $m s$ on individual externally wetted ILIS. Those authors postulated that onset times were likely dominated by viscous forces inherent in the specific emitter geometry and wetting state, rather than charge relaxation for example. Compared to the thin liquid meniscus of externally wetted emitters used in the reference, liquid in the the porous glass emitters used here was located in and channeled through multiple pore orifices near the emitter apex. Viscous effects may therefore have been suppressed, and the liquid may have been more immediately subjected to high electric fields (owning to the absence of a shielding effects due to a metallic emitter). Onset times would therefore have been shorter; however, this hypothesis requires verification. Furthermore, in the devices presented here, 100's of emission sites are likely present over the emitter arrays each making a small contribution towards the total beam current and characterized by an onset field (/voltage) dependent on local conditions. At a single polarity and emitting 100's of $\mu A$, individual emission site onset and collapse is likely to occur during beam current amplitude modulation yet at sufficiently distributed potentials to avoid large, discreet, steps in current[32]. Regardless, minimum onset times may differ between polarities (as observed in Ref. [31]) and emission sites; complicating current following during potential alternations. Recall in Figs. 11 and 12 the floating ground potential was always driven to the imposed limits upon polarity alternations. Thus in addition to improvements in current following circuitry, further study of onset times directed at the specific electro-hydrodynamic conditions of a particular emission source should be pursued to completely assess the feasibility and optimal implementation of charge neutralization in this manner.

The introduced method may never lead to exact current balancing necessitating some reliance on ambient conditions. For example a constant bias error on the order of $1 n A$ in the onboard current summation, equivalent to roughly five parts per million of the beam current in the present case, would lead a spacecraft with $30 \mathrm{pF}$ capacitance to charge at approximately $33 \mathrm{~V} / \mathrm{s}$. Thus although the $\mu A$ level accuracy of monitors used here enabled total-current control at a level two orders of magnitude lower than the beam currents, bias errors may have prevented long-term steady-state charge balancing; even if extractor currents had been actively accounted for. However, active control lends itself to relatively simple customization to suit an environment or mission. A trait which may suppress the degree to which improved current measurement accuracies are required. For example, in an electron-rich LEO environment, the target level for current balance may be adjusted to favour a slightly negative current bias at all times. Positive charging may then be suppressed by collection of an ambient electron current much lower than that of the beam. This is in contrast to a passive system where the spacecraft charge may periodically swing to high negative levels not easily nulled by relatively immobile ambient ions. Conversely in GEO or 
deep-space, a slight positive current balance may enable photo-emission to null resultant negative charging.

It is noteworthy that while numerous electrospray thrusters similar to those shown here could be combined to yield multi-Watt high thrust systems, the tolerable level of net current uncertainty to prevent charging would remain unchanged. The required level of relative current sensing resolution would therefore increase accordingly. At very high power levels approaching kilowatts, consideration of dedicated neutralizers as in electrostatic plasma thrusters may become favourable to offset associated control complexity.

\subsection{Compliance with Electrochemical Charge Balancing}

When operated as a simultaneous bipolar thruster, total currents at any instant must be matched to ensure charge neutralization. Electrochemical balancing through potential alternation seeks to suppress reactions through, similarly, preventing a net charge transfer at the IL to conductor interface[9]. These two balances must be considered in parallel. In doing so it is important to clarify that achieving electrochemical neutralization at a propellant tank does not, inherently ensure beam charge neutrality, and vice-versa. Consider the simple, two source, configuration presented here. Two tanks, and therefore IL to metal electrical contacts, are required to achieve simultaneous bipolar emission. Suppression of electrochemical degradations (of the propellant or electrode) via potential alternation $[9,10]$ is achieved by switching polarity at a rate faster than the time required for the double layer potential to exceed the electrochemical window of the IL-interface. This interfacial potential increases due to the collection of charges of one polarity or another. As previously identified[33, 34], the currents emitted during each half period of operation must match to ensure zero total charge transfer on average. If this condition is not met, charge would gradually accrue over multiple periods that after some time must be dissipated through an electrochemical reaction or other discharge. Such dissipations may occur at a lower rate than if no alternation were employed; however, after the 1000's of hours of operation envisioned for ILIS thrusters, long term propellant stability may necessitate suppression of these integral imbalances.

Thus two constraints exist in a bipolar ILIS propulsion system: i) total beam currents must instantaneously sum to zero to neutralize charge and ii) the average current over potential alternation periods, from each propellant tank, should be nulled. Duty cycle modulations have been employed to satisfy the later requirement for ILs with a propensity to emit higher currents in one polarity compared with another[33]. However; the practical benefits of such a solution are diminished in a bipolar configuration when satisfying constraint i). Thus it is apparent that, at least for a thruster pair, satisfaction of both constraints may be best achieved if both sources emit the same magnitude of current at all times. This subtlety places an additional emphasis on the benefits of high speed, closed loop, current control in bipolar ILIS. Specifically, a relatively slow, current control loop on a single thruster module be used to suppress electrochemical charge buildup over time, while a fast charge neutralization circuit effects accurate mirroring by one or more other modules. Even with such an arrangement currents intercepted by the extractor or other electrodes would compromise the degree to which both of these constraints could be completely satisfied at all times.

\subsection{Multiplexed Bipolar Thrust}

The results of section 3.3, demonstrated that the total thrust output from bipolar modules emitting opposite polarity beams in close proximity was consistent with the sum total of thrust calculated from each module individually. The thrust coefficients $\alpha^{+}$and $\alpha^{-}$in equation 2 were generated using different singlepolarity devices of the same fundamental design as each module in the bipolar devices used here. To emphasize this agreement, Figure 15, presents numerous thrust measurements and calculated thrust levels versus the commanded thrust for two devices. Data in Figure 15(a) were acquired using the same device utilized in measuring the data of Fig. 13 while data in Fig. 15(b) were acquired with another, again nominally identical, dual module device operating with EMI-BF 4 . Directly measured thrusts exhibited greater variability in the latter case yet were often, again, within a few $\mu N$ of calculated total thrust.

As downstream beam neutralization is not fundamentally required for ILIS operation, as evidenced by their typical unipolar operation, the lack of a significant detraction from calculated thrusts was not unexpected. Had the thruster electronics been floated and allowed to charge to many hundreds of volts during thrust measurements, deviations in thrust output would have been expected due downstream acceleration or deceleration of the emitted beams in response to the resultant non-zero electrostatic field at the thruster exit[20]. Although no clear effects were observed here, more thorough investigations of beam neutralization in bipolar ILIS are required to fully comprehend the implications of beam interactions. For example, despite charge neutralization via current balancing, virtual anodes (or cathodes) formed due to poor beam neutralization could lead to accelerated return of low energy species and/or charge-exchange susceptible neutrals born from fragmentation events. Future investigations could include, for example, emissive probes as used to measure colloid and FEEP thrusters in Refs. [35] and [36]. However numerical modelling as performed in Refs. [37, 38] would likely have significant contributions to interpreting experimental data due to the relatively unique nature of the plume with a negligible free electron population. 


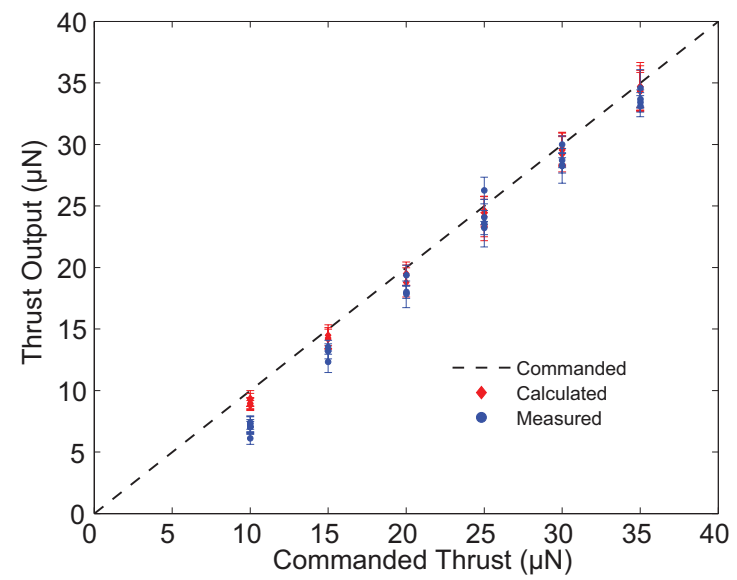

(a)Device 1, used to acquire data in Fig. 13.

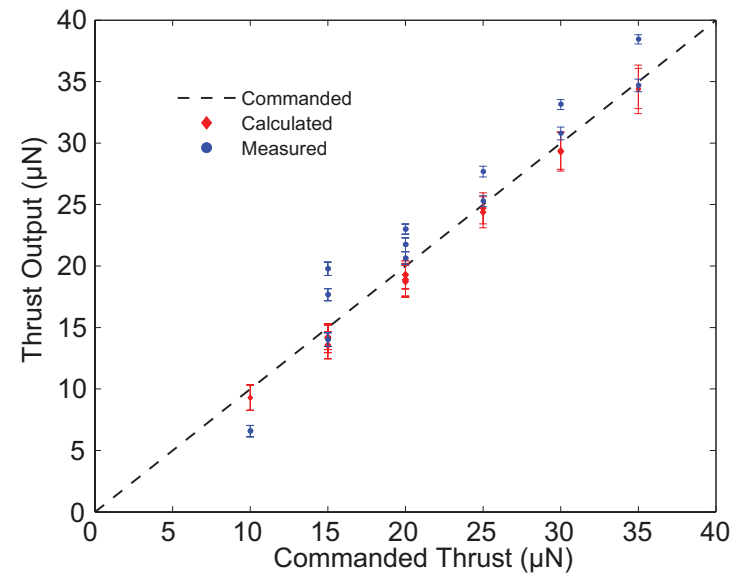

(b)Device 2.

FIG. 15: Compiled directly measured and calculated thrusts from two devices plotted against commanded thrust.

\section{CONCLUSIONS}

Charge neutralization necessitates all currents to and from a spacecraft sum to zero. The degree to which electrostatic ion thruster beams must explicitly comprise zero net current, and the time scales inherent, are dependent on the local space environment. A thruster that is benign to its environment should target charge neutralization by design. Ion-ion charge neutralization using ionic electrospray sources has been discussed and considered experimentally. Two methods for effecting charge neutralization were evaluated experimentally, a natural/passive means and a closed-loop current control circuit.

With no explicit coupling between the emission current governing emitter-to-extractor potential difference and the spacecraft to environment potential, the effectiveness of passive automatic current balancing via a common extractor floated with respect to the thruster common was demonstrated to be limited. Specifically under typical scenarios where extractor currents are neither identically balanced nor negligible, current collection on the extractor grids was shown to lead to rapid charging of thruster common surfaces with respect to an isolated facility ground.

Alternatively, a simple circuit was used to match the magnitudes of positive and negative beam currents at $\sim 10 \mathrm{~ms}$ time scales. This method was shown to be effective at suppressing charge accumulation in some circumstances. However; rapid charging occurred upon beam polarity alternations and differences in extractor current interception fractions between polarities necessitated a polarity-specific manual tuning. In future developments, the former may be partially mitigated through utilizing faster circuitry; modulation up to 450 $\mathrm{Hz}$ was shown to be feasible here. Similarly, a circuit which includes extractor currents in the to-be-nulled current summation may suppress the latter complication.

Two thruster modules, operated in close proximity, were shown to yield a sum total of thrust consistent with previously reported thrust coefficients. Specifically, thrust levels up to $35 \mu N$ were commanded, and measured directly, at up to $0.8 W$ of total input power. No detrimental or performance limiting effects due to beam interactions or a lack of neutralization were observed. However; beam neutralization of ionic electrospray beams requires further study.

\section{Acknowledgments}

This work has been supported through European Space Agency Networking/Partnership Initiative contract \#4000109063/13/NL/PA. The authors would like to thank Dr. Shu T. Lai for his helpful responses to our queries.

\section{References}

[1] Lozano, P. and Martínez-Sánchez, M., "Ionic liquid ion sources: characterization of externally wetted emitters," Journal of Colloid and Interface Sciences, Vol. 282, No. 2, 2005, pp. 415.

[2] Courtney, D. G., Dandavino, S., and Shea, H.,
"Comparing Direct and Indirect Thrust Measurements from Passively Fed Ionic Electrospray Thrusters," Journal of Propulsion and Power, Vol. 32, No. 2, 2015, pp. 392-407.

[3] Krejci, D., Mier-Hicks, F., Fucetola, C., Lozano, 
P., Schouten, A. H., and Martel, F., "Design and Characterization of a Scalable Ion Electrospray Propulsion System," $34^{\text {th }}$ International Electric Propulsion Conference, Hyogo-Kobe, Japan, July 2015.

[4] Gassend, B., Velasquez-Garcia, L. G., M., A. I. A., and Martinez-Sanchez, "A microfabricated planar electrospray array ionic liquid ion source with integrated extractor," Microelectromechanical Systems, Journal of, Vol. 18, No. 3, 2009, pp. 679694.

[5] Legge, R. and Lozano, P., "Performance of Heavy Ionic Liquids with Porous Metal Electropsray Emitters," $44^{\text {th }}$ Joint Propulsion Conference \&6 Exhibit, AIAA2008-5002, Hartford, CT, July 2008.

[6] Dandavino, S., Ataman, C., Ryan, C. N., Chakraborty, S., Courtney, D. G., Stark, J. P. W., and Shea, H., "Microfabricated electrospray emitter arrays with integrated extractor and accelerator electrodes for the propulsion of small spacecraft," Jounal of Micromechanics and Microengineering, Vol. 24, No. 7, 2014, pp. 075011.

[7] Perel, J., Mahoney, J. F., Moore, R. D., and Yahiku, A. Y., "Research and Development of a Charged-Particle Bipolar Thruster," AIAA Journal, Vol. 7, No. 3, 1969, pp. 507-511.

[8] Lozano, P. and Martínez-Sánchez, M., "Studies on the Ion-Droplet Mixed Regime in Colloid Thrusters," Ph.D Thesis, Massachusetts Institute of Technology, Cambridge, MA, 2003.

[9] Lozano, P. and Martínez-Sánchez, M., "Ionic liquid ion sources: suppression of electrochemical reactions using voltage alternation," Journal of Colloid and Interface Sciences, Vol. 280, 2004, pp. 149-154.

[10] Brikner, N. and Lozano, P. C., "The role of upstream distal electrodes in mitigating electrochemical degradation of ionic liquid ion sources," $A p$ plied Physics Letters, Vol. 101, No. 19, 2012, pp. 193504.

[11] Roussel, J.-F., Tondu, T., Matéo-Vélez, J.-C., Chesta, E., D'Escrivan, S., and Perraud, L., "Modeling of FEEP Plume Effects on MICROSCOPE Spacecraft," IEEE Transactions on Plasma Science, Vol. 36, No. 5, 2008, pp. 2378-2386.

[12] Zafran, S., Beynon, J. C., Kidd, P. W., Shelton, H., and Jackson, F. A., "One-mlb Colloid Thruster System Development," Journal of Spacecraft, Vol. 10, No. 8, 1973, pp. 531-533.

[13] Ziemer, J. K., Randolph, T. M., Franklin, G. W., Hruby, V., Spence, D., Demmons, N., Roy, T., Ehrbar, E., Zwahlen, J., Martin, R., and Connolly, W., "Colloid micro-newton thrusters for the space technology 7 mission," IEEE Aerospace Conference 2010, Big Sky, MT, March 2010, also AERO2010-5446760.

[14] Demmons, N., Hruby, V., LaMarre, N., Metivier, E., Ziemer, J., and Parker, M., "Electrosrpay Thruster Propellant Feed System for Gravity Wave Observation Missions," $52^{\text {nd }}$
AIAA/SAE/ASEE Joint Propulsion Conference, Propulsion and Energy Forum, Salt Lake City, UT, July 2016, also AIAA-2016-4739.

[15] Reissner, A., Buldrini, N., Seifert, B., Horbe, T., Plesescu, F., and Scharlemann, C., "The IFM Thruster - Introducing very high $\Delta v$ Capabilities for Nanosats and Cubesats," $52^{\text {nd }}$ AIAA Joint Propulsion Conference, Salt Lake City, UT, 2016, also AIAA-2016-5044.

[16] Lai, S. T., Spacecraft Charging, AIAA American Institute of Aeronautics and Astronautics, 2011.

[17] Garrett, H. B., "The Charging of Spacecraft Surfaces," Reviews of Geophysics and Space Physics, Vol. 19, No. 4, 1981, pp. 577-616.

[18] Garrett, H. B. and Whittlesey, A. C., "Spacecraft Charging, An Update," IEEE Transactions of Plasma Science, Vol. 28, No. 6, 2000, pp. 20172028.

[19] Lai, S., "An Overview of Electron and Ion Beam Effects in Charging and Discharging of Spacecraft," IEEE Transactions on Nuclear Science, Vol. 36, No. 6, 1989, pp. 2027-2031.

[20] Mier-Hicks, F. and Lozano, P. C., "SpacecraftCharging Characteristics Induced by the Operation of Electrospray Thrusters," Journal of Propulsion and Power, 2016.

[21] Courtney, D. G., Li, H. Q., and Lozano, P., "Emission Measurements from Planar Arrays of Porous Ionic Liquid Ion Sources," IOP Journal of Physics $D$, Vol. 45, No. 48, 2012, pp. 485203.

[22] Hutchinson, I. H., "Ion Collection by a Sphere in a Flowing Plasma: 3. Floating Potential and Drag Force," Plasma Physics and Controlled Fusion, Vol. 47, No. 1, 2004.

[23] Hastings, D. E., "A Review of Plasma Interactions with Spacecraft in Low Earth Orbit," Journal of Geophysical Research, Vol. 100, 1995, pp. 1445714483.

[24] Frooninckx, T. B. and Sojka, J. J., "Solar Cycle Dependence of Spacecraft Charging in Low Earth Orbit," Journal of Geophysical Research, Vol. 93, 1992, pp. 2985-2996.

[25] Sasaki, S., Kawashima, N., Kuriki, K., Yanagisawa, M., and Obayashi, T., "Vehicle charging observed in the SEPAC Spacelab-1 experiment," Journal of Spacecraft and Rockets, Vol. 23, No. 2, 1986, pp. 194-199.

[26] Goebel, D. M. and Katz, I., Fundamentals of Electric Propulsion: Ion and Hall Thrusters, John Wiley and Sons, Inc., Hoboken, New Jersey, 2008.

[27] Courtney, D. G. and Shea, H., "Influences of porous reservoir Laplace pressure on emissions from passively fed ionic liquid electrospray sources," Applied Physics Letters, Vol. 107, No. 10, 2015, pp. 103504.

[28] de la Mora, J. F., "The Fluid Dynamics of Taylor Cones," The Annual Review of Fluid Mechanics, Vol. 39, 2007, pp. 217-243.

[29] Courtney, D. G. and Shea, H., "Fragmentation in Time-of-Flight Spectrometry-Based Calculations of Ionic Electrospray Thruster Performance," 
Journal of Propulsion and Power, Vol. 31, No. 5, 2015, pp. 1500-1504.

[30] Lozano, P., "Energy Properties of an EMI-Im ionic liquid ion source," Journal of Physics D: Applied Physics, Vol. 39, 2006, pp. 126-134.

[31] Lozano, P. and Martínez-Sánchez, M., "On the dynamic response of externally wetted ionic liquid ion sources," Journal of Physics D: Applied Physics, Vol. 38, No. 14, 2005, pp. 2371.

[32] Guerra-Garcia, C., Krejci, D., and Lozano, P., "Spatial uniformity of the current emitted by an array of passively fed electrospray porous emitters," Journal of Physics D: Applied Physics, Vol. 49, No. 11, 2016, pp. 115503.

[33] Coffman, C., Courtney, D. G., Hicks, F., Jamil, S., Li, H., and Lozano, P., "Progress Toward a Variable Specific Impulse Electrospray Propulsion System," $47^{\text {th }}$ Joint Propulsion Conference \& Exhibit, San Diego, CA, July 2011, AIAA-2011-5591.

[34] Castro, S. and de la Mora, J. F., "Effect of tip curvature on ionic emission from Taylor cones of ionic liquids from externally wetted tungsten tips," Journal of Applied Physics, Vol. 105, 2009, pp. 034903.

[35] Marrese-Reading, C., Polk, J., Mueller, J., Owens, A., Tajmar, M., Spindt, C., and Fink, R., "In-FEEP Thruster Ion Beam Neutralization with Thermionic and Field Emission Cathodes," $27^{\text {th }}$ International Electric Propulsion Conference, 2001, also IEPC-01-290.

[36] Marrese-Reading, C., Ziemer, J. K., GameroCastano, M., Bame, D., Demmons, N., and Hruby, V., "Plasma Potential Measurments in the Plume of a Colloid Micro-Newton Thruster," $42^{\text {nd }}$ AIAA Joint Propulsion Conference, 2006, also AIAA2006-4042.

[37] Wheelock, A., Cooke, D. L., and Gatsonis, N. A., "Electron-Ion Beam Coupling Through Collective Interactions," $42^{\text {nd }}$ AIAA Joint Propulsion Conference, Sacramento, CA, July 2006, AIAA-065024.

[38] Tajmar, M. and Wang, J., "Three-Dimensional Numerical Simulation of Field-Emission-ElectricPropulsion Neutralization," Journal of Propulsion of Power, Vol. 16, No. 3, 2000, pp. 536-544. 